\title{
Pairwise correlations in cricket cercal interneurons are significant for decoding
} Jean Verrette*, Alex Dimitrov and John Miller

\author{
Address: Center for Computational Biology, Montana State University, Bozeman, MT, USA \\ Email: Jean Verrette* - verrette@cns.montana.edu \\ * Corresponding author
}

from Sixteenth Annual Computational Neuroscience Meeting: CNS*2007

Toronto, Canada. 7-12 July 2007

Published: 6 July 2007

BMC Neuroscience 2007, 8(Suppl 2):PI59 doi:I0.I I86/I47I-2202-8-S2-PI59

(c) 2007 Verrette et al; licensee BioMed Central Ltd.

\section{Background}

To what extent do pairwise correlations exist in sensory systems? Neurons communicate independently, pairwise, or as large populations through features including spike rates and interspike intervals. Previous nonlinear decoders of the acheta domestica cercal system estimated stimuli using responses of single primary afferent neurons and interneurons (Z. Aldworth, T. Ganje: private communication). Here, we develop new decoders based on multi-unit data to quantify the interdependence of the interneuron responses. Our first decoder estimates stimuli from a joint probability distribution. Our second decoder assumes interneurons can be decoded independently. Through the correlation measure $\Delta \mathrm{I}$ developed by Latham et al. [1], we demonstrate that the joint decoder reveals more information about the stimulus than the independent decoder.

\section{Methods}

We present repetitions of white noise sensory stimuli to the preparation and record spike trains extracellulary. After spike sorting, we quantize the times of spikes to create short patterns based on interspike intervals. We approximate marginal, conditional, and joint probabilities of stimuli and responses to measure correlations between pairs of neurons. For the independent interneuron decoder, we calculate the individual stimulus-conditioned response distribution. For the decoder which assumes the interneurons are dependent, we calculate the joint stimulus-conditioned response distribution. With Bayes' theorem, we create an estimate of the joint response and stimulus distributions for each of the two decoders.

\section{Conclusion}

Substantial $\Delta \mathrm{I}$ values indicate significant pairwise correlations in cricket cercal interneurons. Our results imply that decoders which incorporate correlation are needed to understand the processing of sensory stimuli in the cricket cercal sensory system.

\section{Acknowledgements}

The project described was supported by NIH Grant Number P20 RRI6455-06 from the INBRE-BRIN Program of the National Center for Research Resources.

\section{References}

I. Latham P, Nirenberg S: Synergy, redundancy, and independence in population codes, revisited. I Neurosci 2005, 25:5195-5206. 